\title{
Syndromic Craniosynostosis
}

\author{
Christopher Derderian, M.D. ${ }^{1}$ James Seaward, M.B.B.S., M.R.C.S. ${ }^{1}$ \\ ${ }^{1}$ Department of Plastic Surgery, University of Texas Southwestern \\ Medical Center, Dallas, Texas \\ Semin Plast Surg 2012;26:64-75. \\ Address for correspondence and reprint requests Christopher \\ Derderian, M.D., Department of Plastic Surgery, University of Texas \\ Southwestern Medical Center, 1801 Inwood Road, Dallas, TX 75390 \\ (e-mail: Christopher.Derderian@utsouthwestern.edu).
}

\begin{abstract}
Keywords

- syndromic craniosynostosis

- intracranial pressure

- posterior vault distraction

- spring cranioplasty

- midface distraction

Although most cases of craniosynostosis are nonsyndromic, craniosynostosis is known to occur in conjunction with other anomalies in well-defined patterns that make up clinically recognized syndromes. Patients with syndromic craniosynostoses are much more complicated to care for, requiring a multidisciplinary approach to address all of their needs effectively.

This review describes the most common craniosynostosis syndromes, their characteristic features and syndrome-specific functional issues, and new modalities utilized in their management. General principles including skull development, the risk of developing increased intracranial pressure in craniosynostosis syndromes, and techniques to measure intracranial pressure are discussed. Evolving techniques of the established operative management of craniosynostosis are discussed together with more recent techniques including spring cranioplasty and posterior cranial vault distraction osteogenesis.
\end{abstract}

Craniosynostosis can occur as an isolated event resulting in nonsyndromic craniosynostosis, or it can occur in conjunction with other anomalies in well-defined patterns that make up clinically recognized syndromes. Patients with syndromic craniosynostoses are much more complicated to care for, requiring a multidisciplinary team to address all of their needs effectively. These are typically genetic in nature, and may demonstrate autosomal dominant, autosomal recessive, and X-linked patterns of inheritance. Although busy tertiary care centers will encounter a broad range of syndromes, the more commonly identified craniosynostosis syndromes seen by plastic surgeons include Crouzon, Saethre-Chotzen, Apert, Pfeiffer, and Muenke syndromes. These variably share some common features in addition to craniosynostosis including exophthalmos, midface hypoplasia, cranial base anomalies, abnormal facies, and limb anomalies. In fact, the craniofacial features of the various syndromes can be so similar that the digital anomalies may be the sole differentiating physical finding to allow a clinical diagnosis.

Surgery for craniosynostosis dates from the $19^{\text {th }}$ century, but early operations carried high complication rates with poor long-term outcomes. Cranial vault reconstruction did not gain widespread acceptance until 1967 when Paul Tessier revolutionized the field by introducing his intracranial approach that allowed accurate osteotomy, mobilization, and repositioning of the recessed forehead and supraorbital regions. Since that time, several significant advances including surgical intervention in infancy, the advent of computed tomography, introduction of rigid and later resorbable plating systems, and distraction osteogenesis have fueled the evolution of our approach to the treatment of patients with syndromic craniosynostosis. The goal of this article is to review the salient features of the commonly encountered craniosynostosis syndromes and both the traditional and cutting-edge approaches to treatment.

\section{General Considerations}

Craniofacial growth generally follows a craniocaudad pattern with an initial rapid calvarial growth during infancy, followed by orbital and midface growth in the first decade and mandibular growth in adolescence. Over the first year of life, the brain triples in volume to reach two thirds of its adult size. It continues to grow rapidly over the next 2 years, then growth continues more gradually with the brain reaching adult size between 6 and 10 years of age. Skull growth occurs
Issue Theme Craniofacial Surgery; Guest Editor, Edward P. Buchanan, M.D.
Copyright (c) 2012 by Thieme Medical Publishers, Inc., 333 Seventh Avenue, New York, NY 10001, USA. Tel: +1(212) 584-4662.
Dol http://dx.doi.org/ $10.1055 / \mathrm{s}-0032-1320064$. ISSN $1535-2188$. 
both by appositional growth and by the growing brain causing displacement of the overlying cranial bones, which in the presence of patent sutures, drives bone deposition at the suture site in a direction perpendicular to the suture line. The etiology of the craniosynostosis remains enigmatic, but the resulting characteristic patterns of restricted and compensatory growth are well recognized. Although these compensatory growth patterns can be significantly disfiguring, the most concerning consequence of craniosynostosis is the uncertain ability of the developing skull to accommodate the volume requirements of the growing brain. Insufficient skull growth can result in cephalocranial disproportion, which is a significant (but not the sole) factor causing raised intracranial pressure (ICP). ${ }^{1}$ The risk of elevated ICP increases exponentially with the number of affected sutures. In syndromic craniosynostoses, the risk of intracranial pressure can be further elevated due to intracranial venous congestion, hydrocephalus, and upper airway obstruction. ${ }^{1-3}$

The consequences of delayed diagnosis of elevated intracranial pressure can result in optic atrophy, blindness and developmental delay making its detection and prevention a priority in treating patients with craniosynostosis. However, determining whether a child has raised ICP is problematic. The symptoms of frequent worsening morning headache, recurrent vomiting, and an onset of developmental delay may indicate concern for raised ICP; however, they are neither sensitive nor specific enough to be used for a reliable diagnosis. Today, direct intraparenchymal monitoring remains the gold standard, but this approach requires admission to the hospital and it is invasive, carrying risks of bleeding, infection, mechanical failure, and cerebrospinal fluid leak. ${ }^{1}$ Although this modality can give a direct measure of ICP, there is no clear consensus on timing, frequency, and duration of monitoring needed to achieve adequate sensitivity. Similarly, there is no consensus on how to interpret the data because there is no universally accepted scale of normal and abnormal ICP values in children.,4

Proposed noninvasive modalities for monitoring ICP have included radiographs, funduscopic examination for papilledema, transorbital ultrasound, and visual evoked potentials. Papilledema is swelling of the optic disk that occurs in response to raised ICP and screening for its presence via funduscopic examination is rapid and inexpensive. A study of 122 children with craniosynostosis who underwent examination for papilledema followed by ICP monitoring demonstrated that the presence of papilledema was a very specific indicator for raised ICP (98\%), but that it was not sensitive in younger children. ${ }^{5}$ One-hundred percent of those over the age of 8 with elevated ICP had papilledema, but only $22 \%$ of children under the age of 8 who had elevated ICP had papilledema. ${ }^{5}$

"Thumb printing" or "copper beating" on a plain radiograph occurs due to pressure from gyri on the inner table of the calvarium. Although this phenomenon occurs more frequently in children with craniosynostosis and raised intracranial pressure, it is also seen in a large percentage of normal children; therefore, its specificity is inadequate to use as a screening tool. ${ }^{6}$

Transorbital ultrasound can be used to measure optic nerve sheath diameter and has been used in the trauma setting to evaluate for acute changes in ICP. Elevations in ICP affect the space around the optic nerve causing an increase in optic nerve diameter, and elevation above age-controlled diameters can reliably predict elevated ICP in some settings. ${ }^{7,8}$ However, a recent study identifying nerve sheath diameters measured with transorbital ultrasound in patients with craniosynostosis and documented papilledema demonstrated lower sensitivity for detection of elevated ICP than that of funduscopy leaving the utility of this technique in question. $^{9}$

Of the noninvasive modalities, the technique that has shown the most promise for detection of raised ICP is the monitoring of visual evoked potentials. This technique measures the latency time of the averaged encephalographic response to a visual stimulus. Wave latency is measured early in the care of the patient to establish a baseline, which may be abnormal in the setting of craniosynostosis. ${ }^{10}$ Increased prolongation of the latency period is an indicator of axonal injury and correlates with elevated ICP. 1,7,10

All of the above techniques are safe and of variable utility in monitoring the patient's ICP. However, a significant limiting factor is the short length of the observation periods, as transient ICP changes can occur with activity, position, and time of day thus these results can be misleading.

Another frequent sequela of syndromic craniosynostosis is Chiari malformation, a downward displacement of the cerebellar tonsils through the foramen magnum and a common feature observed in patients with craniosynostosis syndromes, present in as many as $70 \%$ of patients with Crouzon syndrome, $82 \%$ with Pfeiffer syndrome, and $100 \%$ with Kleeblattshädel. ${ }^{11-14}$ It can cause noncommunicating hydrocephalus as a result of obstruction of cerebrospinal fluid outflow. It can cause headaches, dizziness, nausea, impaired coordination, muscle weakness, and in severe cases, paralysis. The association between Chiari malformation and craniosynostosis is thought to be an acquired condition that develops early in life secondary to hindbrain growth in an abnormally small posterior fossa and that it may be a consequence of craniosynostosis of the lambdoid or cranial base sutures. ${ }^{12,13}$

\section{Craniosynostosis Syndromes}

\section{Crouzon Syndrome}

Crouzon syndrome is characterized by brachycephaly, shallow orbits with ocular proptosis, midface hypoplasia, and an anterior open bite. A defining characteristic of Crouzon syndrome is that no commonly reported limb anomalies are present. It is caused by mutations in the fibroblast growth factor receptor 2 (FGFR-2) that demonstrate an autosomal dominant inheritance pattern; it is observed in 1 in 25,000 live births making it the most common of the syndromic craniosynostoses. These patients demonstrate a spectrum of phenotypic variability and a commonly encountered scenario is one where a mildly affected and previously undiagnosed parent presents with a more severely affected child.

Bicoronal synostosis is the most common pattern observed, resulting in a brachycephalic head shape, although scaphocephaly, trigonocephaly and even the cloverleaf skull deformity have been described. Fusion of the cranial base 


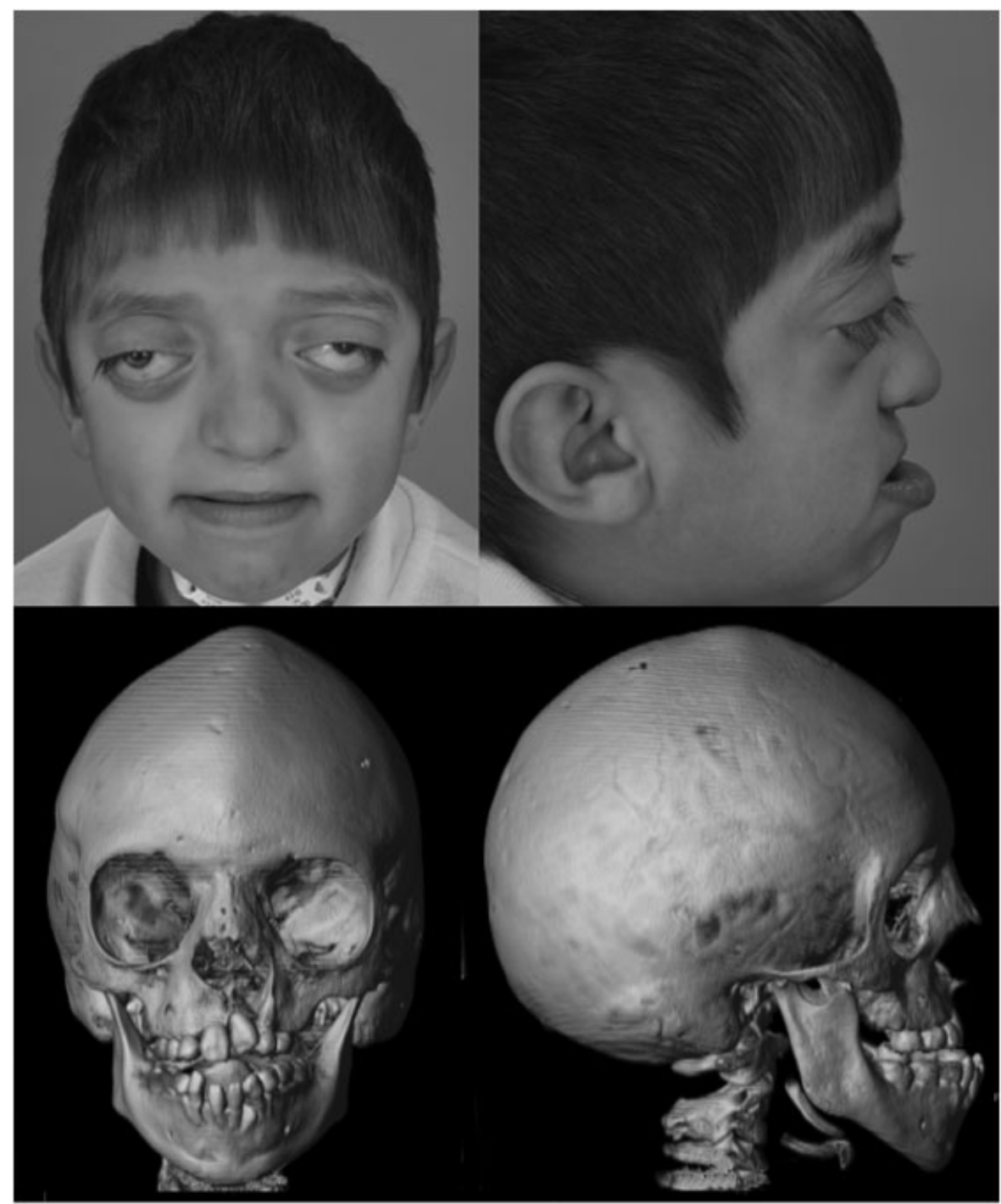

Figure 1 A 6-year-old boy with untreated Crouzon syndrome. The photographs demonstrate exorbitism and midface hypoplasia and the threedimensional computed tomography reconstructions demonstrate pancraniosynostosis and an anterior open bite.

sutures causes shallow orbits with exorbitism; midface hypoplasia; a constricted, high arched palate; and an anterior open bite. The exorbitism is a constant finding and can result in exposure conjunctivitis, keratitis, visual acuity problems, and even herniation of the globe, requiring immediate reduction. The combination of midface hypoplasia with normal mandibular growth leads to a class III malocclusion (-Fig. 1). These patients are typically of normal intelligence. Other associated findings include a conductive hearing deficit, strabismus, and hydrocephalus.

Several reports have identified Crouzon syndrome as carrying a higher risk of elevated ICP than other forms of syndromic craniosynostosis, with one study demonstrating a $65 \%$ incidence of elevated ICP and the remainder borderline elevated. ${ }^{15-17}$ A retrospective review of suture patency in patients with syndromic craniosynostosis may offer some insight. The review demonstrated that patients with Crouzon syndrome demonstrated earlier closure of the lambdoid and sagittal sutures (median 6 and 21 months, respectively) and a $72 \%$ incidence of type I Chiari malformation as compared with patients with Apert syndrome, who demonstrated later suture closure (51 and 60 months, respectively) and a $2 \%$ incidence of type I Chiari malformation. ${ }^{13}$ We agree with others that these clinically significant characteristics are likely related and should be considered when planning the sequence for surgical treatment of these patients. ${ }^{12,18,19}$

\section{Apert Syndrome}

Apert syndrome is characterized by turribrachycephaly, midface hypoplasia, and symmetric syndactyly of both hands and feet. It is caused by mutations in FGFR- 2 that occur in $\sim 1$ in 100,000 births and, although it carries an autosomal dominant inheritance pattern, the majority of cases seen are sporadic new mutations. The most common presentation is bicoronal synostosis, with a characteristic large anterior fontanelle, bitemporal widening, and occipital flattening. These patients have more severe midface hypoplasia than Crouzon patients with concavity of the midface, shallow orbits with mild hypertelorism and downslanting palpebral fissure, ocular proptosis, a high arched or cleft palate, anterior 


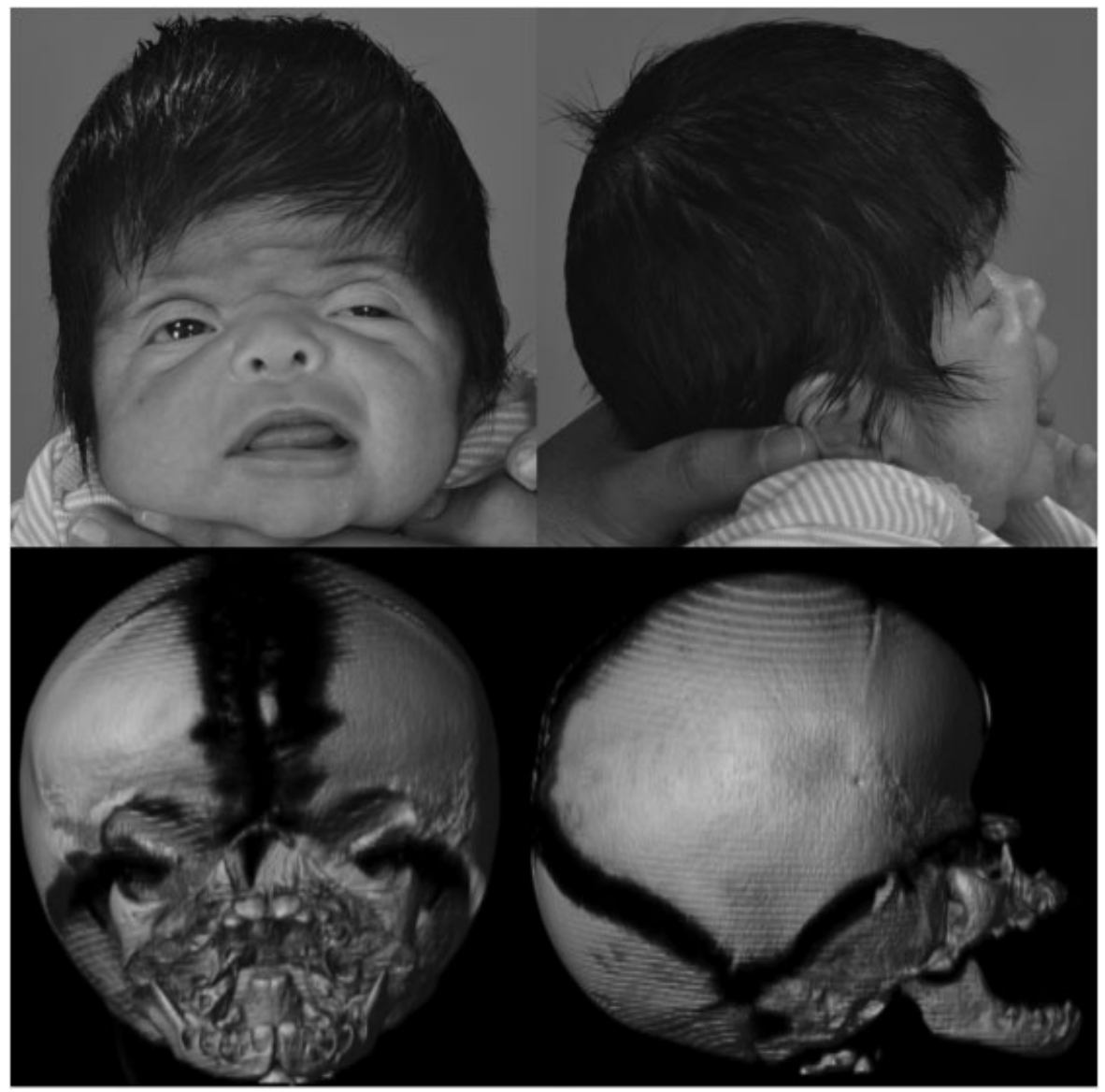

Figure 2 A 1-month-old girl with Apert syndrome. The photographs demonstrate midface concavity, downslanting palpebral fissures, and turribrachycephaly. The three-dimensional computed tomography reconstructions demonstrate partial bicoronal craniosynostosis with a large anterior fontanelle and bitemporal widening.

open bite, and characteristic nasal deformities including a depressed nasal bridge and downturned tip resulting in a socalled parrot beak deformity (-Fig. 2).

The maxillary hypoplasia results in a significant class III malocclusion and commonly results in airway compromise significant enough to warrant tracheostomy. The hand syndactyly, which is pathognomonic for the condition, most often involves fusion of the second, third, and fourth fingers, resulting in middigital hand mass, but the first and fifth fingers may also be joined to the middigital mass. When the thumb is free, it is broad and deviates radially. In the feet, the syndactyly also usually involves the second, third, and fourth toes ( - Fig. 3). These hand anomalies are so severe and functionally debilitating that referral to a hand surgeon with special expertise in this area is essential. These patients carry an increased incidence of delayed mental development, but
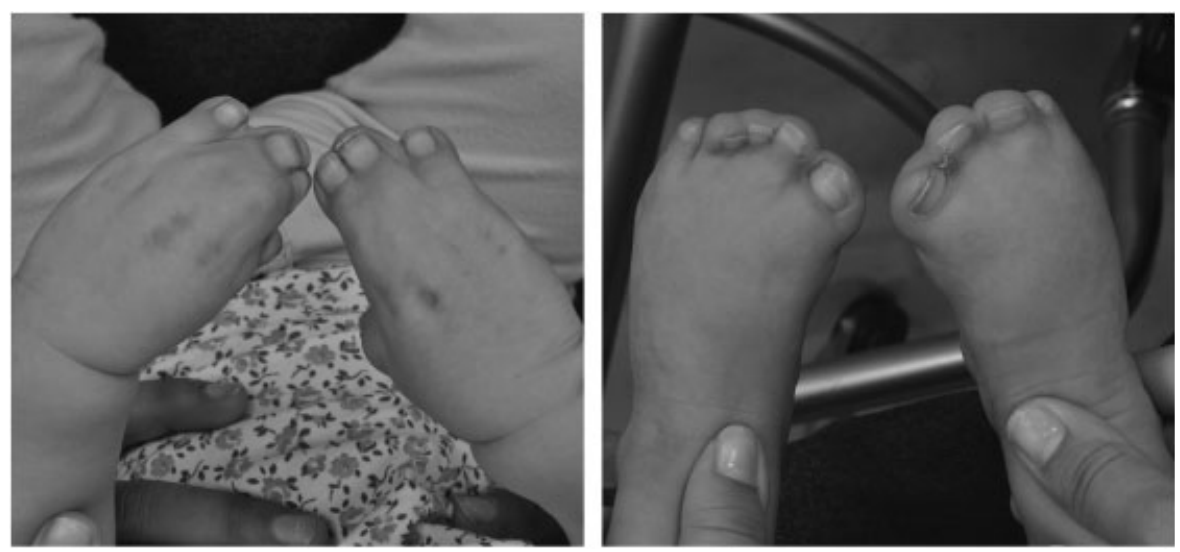

Figure 3 These photographs of the hands and feet of a 1-month-old girl demonstrating the complex syndactyly associated with Apert syndrome. 

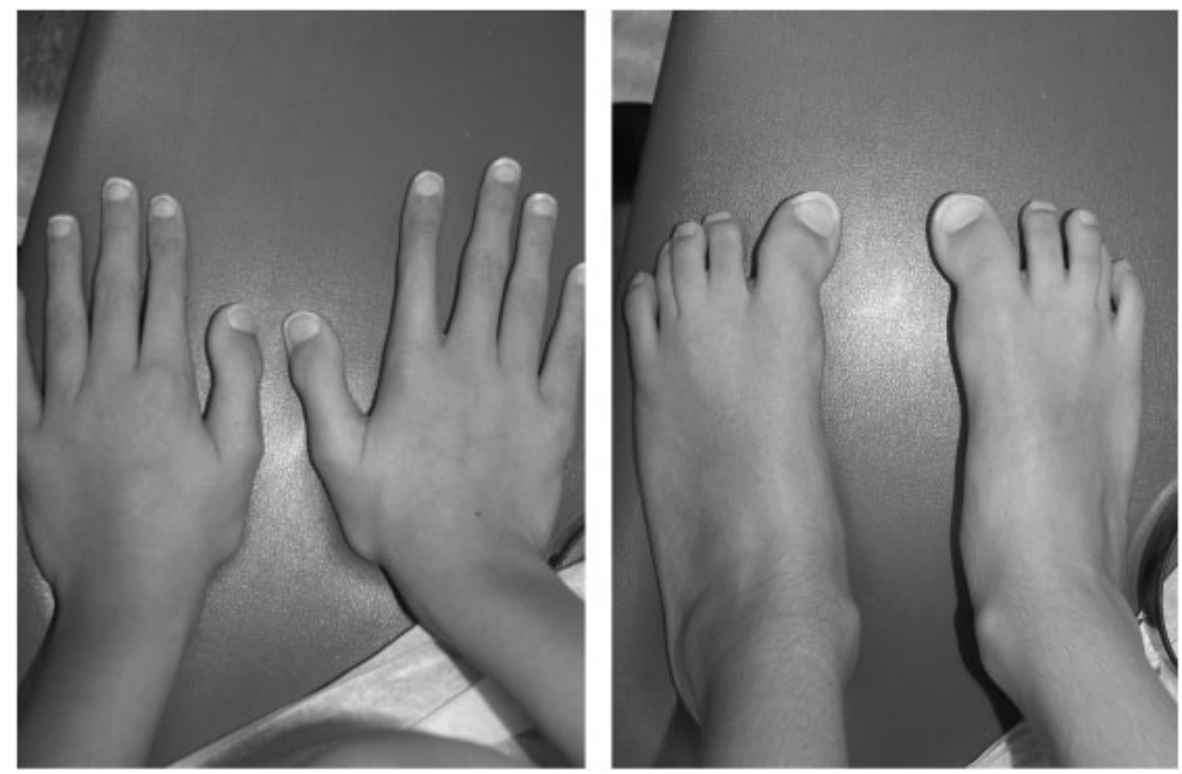

Figure 4 Broad thumbs and great toes, and partial second and third toe syndactyly associated with Pfeiffer syndrome.

many of these patients develop normal intelligence. Acne vulgaris is another characteristic feature seen during adolescence in over $70 \%$ of patients.

Perhaps our best understanding of the natural history of raised ICP in Apert syndrome comes from Marruci et al who published the Great Ormond Street Hospital data on the expectant management of their patients. Their protocol is to offer cranial vault expansion only in the setting of confirmed elevation of ICP. ${ }^{4}$ Raised ICP developed in 83\% (20/24) of patients, $50 \%$ in the first year of life, with the average age of onset at 18 months (range 1 month-4 years 5 months). ${ }^{4}$ Thirty-five percent of those who were treated successfully for their first episode of elevated ICP went on to develop a second episode on average 3 years 4 months later. ${ }^{4}$

\section{Pfeiffer Syndrome}

Pfeiffer syndrome consists of a spectrum of craniofacial features ranging in severity from mild to severe. The most common features include turribrachycephaly, midface hypoplasia, exorbitism, and the hallmark broad thumbs and great toes and variable soft tissue syndactyly (-Fig. 4). Other features include hypertelorism, strabismus, downslanting palpebral fissures, class III malocclusion and a beaked nasal deformity. The majority of cases of Pfeiffer syndrome involves FGFR-2; however, roughly $5 \%$ of patients express an FGFR-1 mutation and demonstrate a less severe phenotype. ${ }^{20,21}$ The incidence is 1 in 100,000 live births and the inheritance pattern is autosomal dominant. Cohen proposed a classification system that clusters patients into three types based upon their clinical findings and severity. Type I represents the classic Pfeiffer syndrome (features described above). Type II is more severe and is associated with a Kleeblattschädel (cloverleaf skull). Type III Pfeiffer syndrome is the most severely affected. ${ }^{22} \mathrm{~A}$ recent review of 28 patients treated at a single institution reported that the Cohen subtypes distribution was $61 \%$ type I, $25 \%$ type II, and $14 \%$ type III. $^{11}$
These patients underwent an average of 2.5 cranial vault procedures, 1.6 neurosurgical procedures, and 3.5 other operations. ${ }^{11}$ In addition to the challenging reconstructive needs of the patient with Pfeiffer syndrome, this study highlighted several functional considerations that should be aggressively treated or monitored in these patients including value in early placement of permanent tarsorrhaphies and supplementing these with temporary tarsorrhaphies at the time of cranial vault procedures, as well as high incidences of aural atresia (54\%), conductive hearing loss (86\%), need for tracheostomy (61\%), hydrocephalus (68\%), and Chiari malformations (82\%). ${ }^{11}$

\section{Saethre-Chotzen Syndrome}

Saethre-Chotzen syndrome occurs in 1 in 25,000 to 50,000 births and is characterized by a wide range of phenotypes and severity caused by the autosomal dominant inheritance of mutations of the TWIST- 1 gene in chromosome $7 \mathrm{p} 21.2 .^{23-25}$ The variability in presentation is highlighted by the heterogeneous pattern of craniosynostosis observed with the majority of patients presenting with bicoronal synostosis (45-76\%); however, unicoronal (18-27\%) and other multisuture presentations are also common (6-18\%). ${ }^{25-28}$ Other defining features that are found in the majority of patients are a low frontal hairline, eyelid ptosis, facial asymmetry, and ear deformities with a characteristic prominent crus helicis extending through the conchal bowl. ${ }^{25-28}$ A minority of these patients will have hearing loss, brachydactyly, syndactyly, or clinodactyly. ${ }^{25,27}$ Midface hypoplasia is not a common feature of Saethre-Chotzen syndrome.

Patients with Saethre-Chotzen have a high incidence of need for reoperation after cranial vault expansion, ranging from 42 to $65 \%{ }^{25,26,29}$ Several reports have demonstrated high reoperation rates for poor growth after frontoorbital advancement; however, a recent report demonstrated that this patient population also carries a greater than $40 \%$ risk of 
developing elevated ICP after the initial cranial vault expansion. ${ }^{25,26,29,30}$ Clearly such a high-risk population warrants strict monitoring and their families should be made aware that the majority of these patients require more than one cranial vault procedure in the course of their treatment.

\section{Muenke Syndrome}

Unlike other eponymous craniosynostosis syndromes, Muenke syndrome derives its name from the first report of the genetic mutation rather than phenotype. The mutation is a pro250Arg mutation in FGFR-3 on chromosome $4 \mathrm{p},{ }^{31}$ which has an incidence of 1 in 10,000 and demonstrates an autosomal dominant inheritance pattern with variable expressivity. ${ }^{31-35}$ It is estimated that Muenke syndrome may be present in $10 \%$ of unicoronal or bicoronal synostosis cases that were previously believed to be nonsyndromic in origin. The most consistent features include craniosynostosis of the coronal sutures, hearing loss, developmental delay, and thimble-like middle phalanges. ${ }^{31,36}$ Midface hypoplasia is not a common finding. ${ }^{32,36}$ Muenke syndrome exhibits significant variability in the presentation of craniosynostosis between genders, where $88 \%$ of females and $76 \%$ of males with the mutation have craniosynostosis. ${ }^{36}$ Although bicoronal synostosis is the most common presentation for both sexes, males demonstrate a much higher incidence of unicoronal craniosynostosis ( $37 \%$ bicoronal vs $29 \%$ unicoronal) than females ( $58 \%$ bicoronal vs $20 \%$ unicoronal). The pattern of sensorineural hearing loss found in these patients is characteristically a bilateral, symmetric low-to-mid frequency pattern. ${ }^{32,36}$

The clinical relevance of Muenke syndrome lies in the course of these patients after their initial surgical treatment. In a large retrospective review of the reoperation rate for patients with coronal craniosynostosis, the reoperation rate for elevated ICP in Muenke syndrome was five times more common than those without the mutation. ${ }^{37}$ Others have also found rates of reoperation to be much higher and aesthetic outcomes to be poorer in Muenke syndrome. ${ }^{32,38}$ In a patient population that is already at an increased risk for developmental delays and lack significant extra cranial signs of their genetic diagnosis, a high level of suspicion and low threshold for genetic testing must be had when evaluating patients with unicoronal or bicoronal synostosis, particularly those with a family history.

\section{Surgery for Syndromic Craniosynostosis}

\section{Surgery Under 1 Year of Age}

The goals of surgical treatment in the first year of life are to increase the intracranial volume, with the aim of reducing the risk of developing elevated ICP and to improve head shape. The techniques most commonly employed for the initial cranial vault expansion are frontoorbital advancement (FOA) with anterior cranial vault remodeling or posterior cranial vault expansion, although other foci for expansion may be employed in specialized circumstances. ${ }^{39}$ Both approaches have traditionally been performed as a single-stage procedure; however, techniques such as cranial vault distraction and spring-assisted cranioplasty are now finding a primary role with the promise of augmenting the change that we can achieve in head shape and intracranial volume with less morbidity. The time to initiate elective surgical treatment of craniosynostosis is controversial and is in large part dictated by the temporal changes in the physical properties of the calvarium in infancy. Before 6 months of age the cranial bones are extremely malleable, but they are too weak to effectively support rigid fixation. Conversely, after 12 months of age, the bones are thicker and more rigid, which facilitates fixation but limits our ability to reshape them. In addition, the innate ability of the infant skull to spontaneously heal large bony defects is lost between 9 and 11 months of age. ${ }^{40}$ Taking all of these factors into consideration, our current approach is to perform open vault remodeling between 6 and 9 months of age. In cases where elevated ICP is suspected or confirmed, urgent decompression with strip craniectomy will be performed in the very young patient (age $<3$ months), with spring-assisted cranioplasty in selected cases between 3 and 6 months or through a standard singlestage or distraction technique from age 6 months on.

At our institution the decision about which technique to employ in the initial vault expansion is determined by the deformity. If ocular exposure issues are present from poor supraorbital rim positioning, then a FOA is performed, otherwise a posterior vault expansion is undertaken. The advantages of posterior cranial vault expansion have been highlighted in numerous publications. ${ }^{12-14,41,42}$ Expansion of the posterior vault provides a larger volume increase per millimeter of advancement than anterior expansion (Derderian et al, unpublished data, 2012). ${ }^{18}$ Many of the patients we treat with syndromic craniosynostosis have turribrachycephaly and occipital flattening, which can be particularly challenging to address in a single stage. Posterior expansion is an ideal focus for surgical intervention in these patients, as it allows for volume expansion to help minimize the risk of, or treat elevated ICP, while providing a significant improvement in head shape. Improvement in head shape is a result of both direct remodeling and providing room for brain growth posteriorly with subsequent reduction in compensatory vertical growth. An additional benefit of posterior vault expansion for patients with syndromic craniosynostosis is the decompressive effects it has in patients with Chiari malformation, a very common finding in this population. ${ }^{12}$ Unfortunately, posterior cranial vault remodeling in the young patient has historically carried the disadvantages of increased blood loss compared with anterior procedures, limitations of advancement due to soft tissue coverage, and a propensity for relapse due to the weight of the head on the posterior skull when the child is lying supine.

\section{Posterior Vault Distraction}

The advantages of distraction osteogenesis have been highlighted by its application in the cranial vault, midface, and mandible including the maintenance of bone vascularity, production of vascularized bone, limiting production of dead space, and gradual expansion of the soft tissue envelope that allows for greater advances to be achieved and maintained in the jaws. ${ }^{41,43-46}$ Disadvantages of distraction include the 

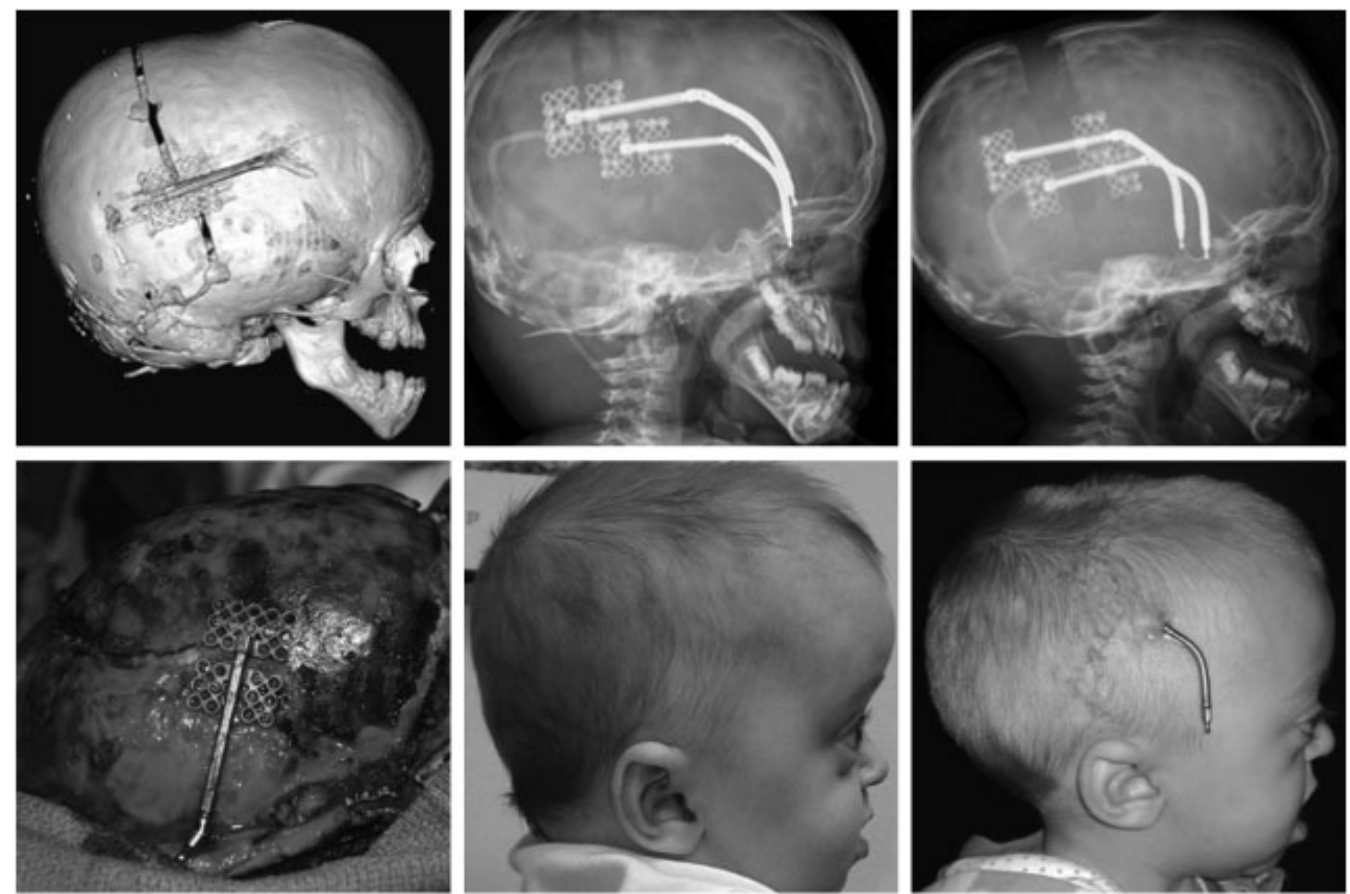

Figure 5 This figure demonstrates distraction osteogenesis of the posterior cranial vault. The left images are three-dimensional computed tomography and intraoperative views of the osteotomies and distraction device placement. The middle images are radiographic and clinical views prior to distraction; the right images are the same views at the end of the activation phase of distraction osteogenesis. (Reprinted with permission from Steinbacher DM, Skirpan J, Puchala J, et al. Expansion of the posterior cranial vault using distraction osteogenesis. Plast Reconstr Surg 127: 792-801).

need for a second procedure for device removal, potential for device-related complications, and prolonged treatment time.

Distraction has been used to address the frontoorbital region in craniosynostosis for over a decade; however, it is only recently that its application to the posterior vault in syndromic craniosynostosis has been described. ${ }^{41,42,47}$ This approach is particularly advantageous in patients with severe turricephaly and occipital flattening as posterior expansion allows for a significant expansion of the intracranial space and improvement in head shape (-Fig. 5). The initial report of the procedure reports some potential improvement in the appearance of even the anterior vault which was untouched, although no quantitative data was presented to support this observation. ${ }^{42}$ Another interesting benefit of distracting the posterior vault is the remarkable improvement observed in the cerebellar anatomy of patients with syndromic craniosynostosis and

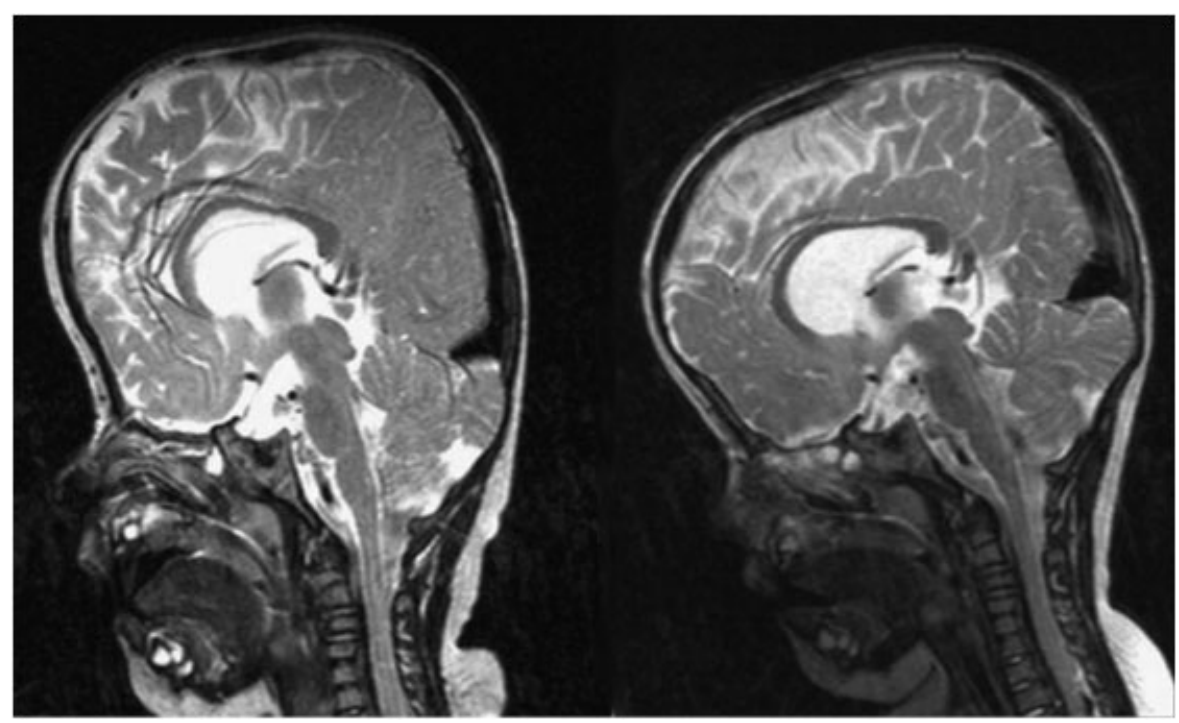

Figure 6 Magnetic resonance images showing a reduction of tonsillar herniation and a more normal cerebrospinal fluid distribution preoperatively on the left and 3 months postoperatively on the right. (Reprinted with permission from White N, Evans M, Dover MS, et al. Posterior calvarial vault expansion using distraction osteogenesis. Child's Nervous System 25, 231-236). 
Chiari malformation ( - Fig. $\mathbf{6}$ ), a frequent finding in syndromic craniosynostosis. $^{12,13,42}$ PVD in these patients appears to have as significant effect on the cerebellum as a traditional decompression procedure; however, a side-by-side comparison of the two techniques has not been published to date. Perhaps the most significant advantage of distracting the posterior over the anterior vault is the simplicity of the anatomy. The posterior vault is more forgiving with a large surface area for distribution of tension and irregularities easily hidden by hair. The posterior vault has fewer aesthetically critical bony features, whereas anteriorly one has to account for the orbital volumes, cornea to orbital rim relationships, and facial proportions and harmony. Thus, PVD allows for greater

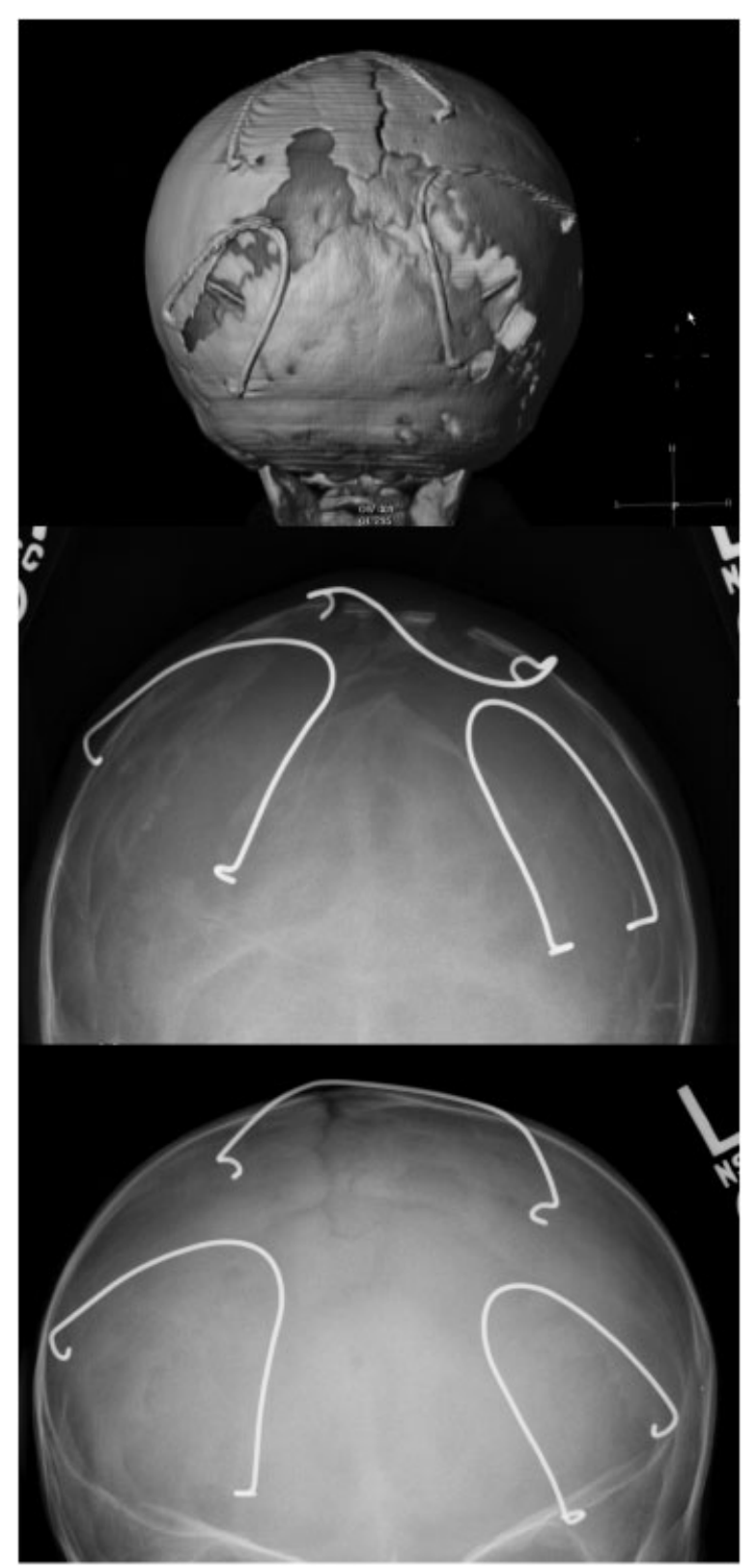

Figure 7 This girl with Apert syndrome underwent spring cranioplasty of her posterior scalp. The top image demonstrates the position of the springs on the skull, the middle radiograph shows the spring position immediately following placement, and the lower radiograph shows the spring position 6 months later. movements, certainly more so than distraction in the anterior vault and supraorbital region. Advancements of over $30 \mathrm{~mm}$ and volumetric gains of over two times those of a single-stage FOA are routine (Derderian et al, unpublished data, 2012). ${ }^{41}$ The long-term durability of these procedures and the ability of the distracted calvarium to grow are unknown to date. However, it will be interesting to see how maintenance of the bone flap vascularity impacts growth and retention of the overcorrection in the long term. Certainly the added stability of the rigid fixation afforded by the distraction devices during the distraction and consolidation phases withstands the propensity for relapse created by the weight of the head while in the supine position. PVD may provide such protection to the growing brain that FOA can be delayed until an older age, perhaps even making a monobloc procedure the first frontoorbital procedure; however, long-term growth and functional data are not yet available.

The procedure is performed with the patient in a prone position. A bicoronal incision is used for access, the scalp reflected, and limited dural dissection is performed to allow a posterior craniotomy and barrel staving at the base of the occiput that prevents a step-off deformity. Two collinear 1.5-mm mandibular distraction devices are applied with a posterior or posteroinferior vector, depending on skull morphology, and the scalp is closed. Activation starts at the end of a 5- to 7-day latency period and proceeds at $1 \mathrm{~mm} / \mathrm{d}$ to reach advancements of 20 to $30 \mathrm{~mm}$. After a consolidation period of 6 to 8 weeks, a limited procedure is required for device removal.

\section{Spring-Assisted Cranioplasty}

Spring-assisted cranioplasty (SAC) uses continuous force generated by a spring across either an osteotomy or a patent suture to achieve a change in head shape and expand the intracranial volume. It is most commonly used in sagittal suture synostosis; however, Lauritzen and others advocate for its use in any of the symmetric patterns of craniosynostosis including syndromic craniosynostosis. ${ }^{48,49}$ It is optimally employed at younger ages when the cranial bones and scalp are most pliable. The morbidity associated with spring-assisted technique is less than that of open procedures, with shorter operative times; however, it does require a second procedure for device removal. ${ }^{50}$ Even though a constant force is applied across the osteotomy or suture with this technique, the surgeon has no control over the distance or rate of advancement at which bones are separated from one another. The expansion is limited only by the equilibrium in forces between the spring and the opposing bones and scalp that are reached rapidly; thus, this is not a form of distraction osteogenesis. Regardless, this is purely a technical note as infants less than 6 months of age can have large bony defects closed with good-quality bone. Despite several reports of favorable outcomes from the use of spring-assisted techniques, this technique remains somewhat controversial. In particular, one must consider the control and stability of expansion when planning SAC in the posterior vault to account for the additional opposing force added by the weight of the infant skull. - Figure 7 clearly demonstrates significant expansion across the patent sagittal and lambdoid sutures after SAC in the posterior vault. 


\section{Frontoorbital Advancement}

The surgical goals of a frontoorbital advancement (FOA) are to expand intracranial volume, reshape the cranial vault, and advance the retruded supraorbital bar to improve globe protection and aesthetic appearance. The patient is placed supine and a bicoronal approach is used. A frontal craniotomy is performed, the frontal bone flaps are raised, and the bandeau is harvested. Early approaches in FOA relied heavily on tongue-in-groove techniques to provide stability to constructs fixed with wire or suture alone. With the advent of absorbable plating systems and the added stability they afford, we feel that the additional dissection and commitment to brow position made with tongue-in-groove techniques are unnecessary and may limit freedom in positioning of the construct; however, this is surgeon dependent. We originate our bandeau cuts at the level of the posterior limit of the craniotomy ( $\mathbf{- F i g . ~ 8 )}$ ). Maneuvers are performed to shape the bandeau, which is advanced, bolstered forward with a cranial bone graft, and secured with absorbable plates. In general, at younger ages of intervention we attempt to overcorrect to the maximal anterior position that can be safely afforded by the soft tissue envelope. Given the impaired AP growth observed with syndromic craniosynostosis affecting the coronal sutures, we find that overcorrection is never a problem. We typically replace the frontal bone flaps in an orthotopic position; however, we will use the orientation that provides the desired brow contour and best accommodates the contour of the advanced bandeau. Once we have committed to frontal bone flap orientation, we harvest cranial bone grafts from the posterior edges of the frontal bone flaps to support the advanced bandeau at the posterior edge of the temporal limbs, which are both secured with resorbable plates. We also place calvarial bone graft struts at the level

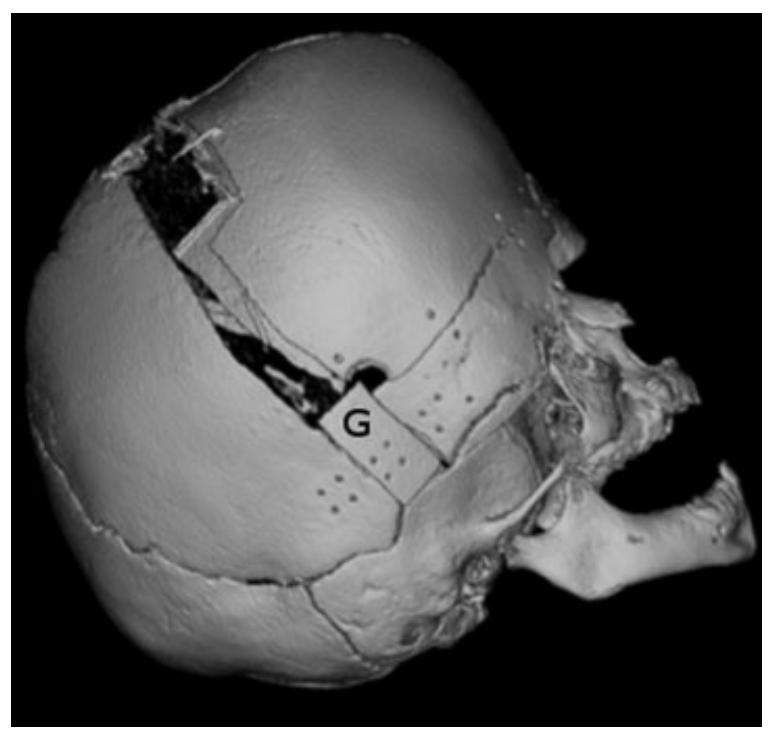

Figure $\mathbf{8}$ This three-dimensional computed tomography reconstruction demonstrates the frontoorbital bandeau, cut at the posterior level of the osteotomy, bolstered forward with bone graft. The graft is marked " $G$ " and the posterior end of the bandeau lies immediately anterior to the graft. of the orbital roofs to provide added stability to the advanced supraorbital bar segment of the bandeau.

Anterior cranial vault remodeling technique is dependent on the preoperative head shape. For severe turricephaly, a total cranial vault reshaping can be performed that allows for a significant reduction in the vertical height of the skull. Patients with less severe turricephaly, as often seen after PVD, typically require only the anterior two-thirds of the vault remodeled.

In patients age 12 months or younger, the majority of the cranial gaps created by FOA will close spontaneously; however, roughly $20 \%$ of all patients undergoing FOA have persistent calvarial defects requiring cranioplasty. ${ }^{51}$ In our experience, these defects are typically small and are of no significant consequence from a safety standpoint, but they remain a source of concern for parents and patients and may cause minor contour irregularities. ${ }^{52}$ We have therefore adopted the practice of primary grafting of these defects in all patients with particulate bone harvested from the endocortex of the frontal bone flaps. ${ }^{51}$

Although FOA and PVD provide excellent volume expansion and improvement in head shape, continued growth restriction in the cranial vault often produces poor long-term functional and aesthetic results in these syndromic patients. If signs of increased ICP, symptoms from eye exposure due to corneal supraorbital rim relationships or abnormal head shape recur, a second and occasionally a third cranial vault expansion procedure may be indicated.

\section{Surgery in Midchildhood}

The timing of midface correction remains controversial among craniofacial surgeons. Some craniofacial centers advocate early surgical correction between the ages of 4 and 7 years, whereas others prefer to wait until full skeletal maturity is reached unless airway obstruction or severe exorbitism warrant early intervention. Advocates for delayed correction cite evidence of a high incidence of recurrent class III malocclusion in patients who undergo surgery earlier, often requiring a secondary procedure in the teenage years. Proponents of early correction believe that the psychosocial benefit of early correction of the patient's midface deformity affords the patient an improved quality of life, to the point where the early procedure is justified even though the second procedure will be required. In our counseling, we advise patients to wait until at least 5 years or as long as there is no negative consequence for the psychological well-being of the child and that they may require a secondary procedure as a standard step in their treatment. In our experience, although the majority of patients are willing to accept this, distraction has made this a less common requirement.

Midface advancement is achieved by performing subcranial osteotomies at the LeFort III level, achieving craniofacial disjunction, and advancing the midface in either a single stage or by distraction osteogenesis. The midface can be advanced alone or in combination with the supraorbital bar and frontal bone flaps as a monobloc procedure. When the correct supraorbital 


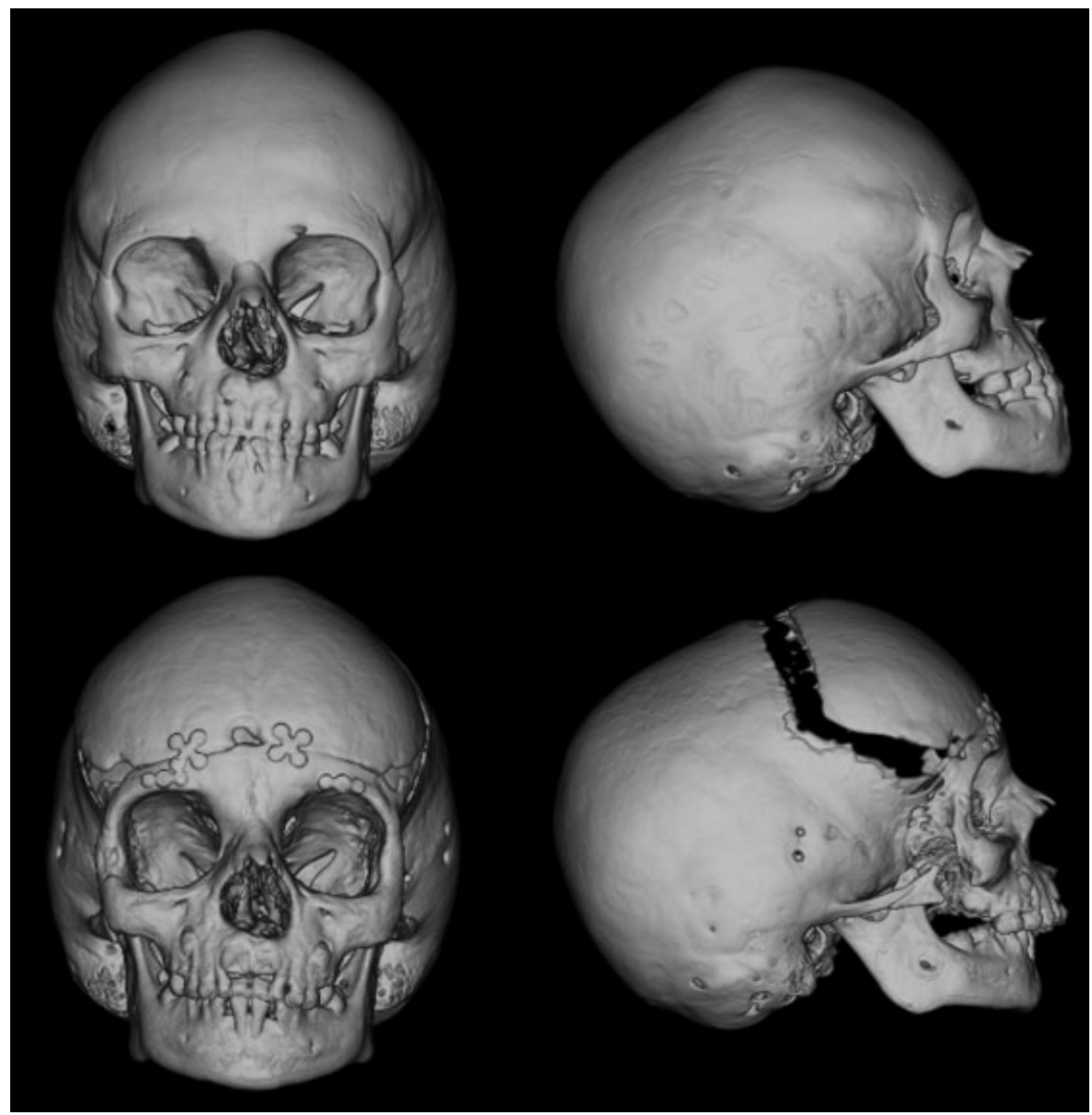

Figure 9 These three-dimensional computed tomography reconstructions demonstrate a monobloc distraction. The top images are anteroposterior and lateral views preoperatively and the lower images are the same views following postconsolidation.

rim-to-cornea relationships are present, the monobloc procedure allows for simultaneously correcting the brow, supraorbital, and midface deformities ( $\mathbf{- F i g . 9}$ ). However the traditional monobloc procedure carries a significant surgical morbidity. Patients with ventricular shunts have an exceedingly high rate of infection because the brain is unable expand into the newly created dead space. The advantages of distraction osteogenesis over single-staged procedures were highlighted earlier, and the decreased morbidity that monobloc distraction affords over the single-stage procedure epitomizes these. ${ }^{44}$ In the era of distraction, the one-stage monobloc procedure should not be performed in most circumstances.

Regardless of the timing of midface advancement, distraction has evolved as the treatment modality of choice for the extensive midface advancement these patients require. The efficacy and improved safety profile of both LeFort III and monobloc distraction over single staged procedures have been well documented. ${ }^{43,44,46,53}$ Distraction allows for expansion of the soft tissue envelope with the bony advancement, decreasing relapse from soft tissue contraction. The procedure involves a standard LeFort III or monobloc osteotomy without acute advancement and placement of an external or semiburied distractor. The surgical technique and distraction protocol is dictated by the patient's bony defor- mity. In addition to LeFort III osteotomy, a facial bipartition may be incorporated to correct the hypertelorism, downslanting palpebral fissures, and midface concavity that are characteristic of Apert and Pfeiffer syndromes. ${ }^{54}$ Although the goals of midface distraction in this age group are tailored to each patient's deformity, the universal goals are maximal projection of the zygoma and restoration of orbital volume. The result is typically a transition from a severe class III to class II malocclusion. The final occlusion is addressed with the definitive orthognathic procedure once the patient reaches skeletal maturity, and occlusion should play little-to-no role in the planning of these procedures. Advantages of distraction include (1) less blood loss and shorter operative time at the initial procedure, (2) greater advancement (up to $20 \mathrm{~mm}$ or more) as compared with standard advancement techniques (6-10 mm maximum), (3) elimination of bone grafts as new bone forms at the osteotomy sites, (4) reduced infection risk with the monobloc procedure, and (5) less relapse. ${ }^{43,44,46,53}$ Disadvantages include the prolonged time needed for distraction and consolidation, a second procedure to remove buried devices, and the requirement of an external halo device for a prolonged period. Overall, distraction osteogenesis has significantly improved the results obtainable for midface advancement while minimizing the complications. 


\section{Surgery in Adolescence}

\section{Orthognathic Surgery}

The abnormal patterns of facial growth in children with craniosynostosis syndromes often result in significant dentofacial deformities. Class III malocclusion, secondary to midface hypoplasia, is the most commonly seen deformity and often develops despite appropriate midface surgical treatment. The team approach to the management of these jaw abnormalities involves an orthodontist, a dentist, and a craniofacial surgeon. Following the completion of growth of both the maxilla and the mandible, surgery may be indicated. This would be preceded by orthodontic therapy to optimize the bite. Surgery is customized to the individual jaw discrepancy, but most commonly involves osteotomy at the LeFort I level with a sliding genioplasty. These surgical procedures are usually performed between the ages of 14 and 18 years, when the facial skeleton is mature.

\section{Final Facial Contouring}

At the completion of facial growth and all major osteotomies, contour irregularities of the facial skeleton may still remain. Final contouring procedures are often performed at this time. They include smoothing down irregularities, adding bone grafts or bone substitutes to different areas (e.g., calcium carbonate cements), and resuspending soft tissues such as the midface or canthus.

\section{Conclusion}

The craniosynostosis syndromes consist of a spectrum of clinical problems with common themes in pathologic findings, treatment strategies, and expected difficulties. Intimate knowledge of the natural history, characteristic findings, and clinical problems of each syndrome are mandatory. The ideal setting to care for these patients is in tertiary care centers with multidisciplinary teams dedicated to such complex problems.

\section{Acknowledgments}

The authors would like to thank Dr. Alex Kane, Professor and Vice-Chairman of the Department of Plastic Surgery at UT Southwestern Medical Center for his clinical and CT images.

\section{References}

1 Tamburrini G, Caldarelli M, Massimi L, Santini P, Di Rocco C. Intracranial pressure monitoring in children with single suture and complex craniosynostosis: a review. Childs Nerv Syst 2005; 21(10):913-921

2 Gonsalez S, Hayward R, Jones B, Lane R. Upper airway obstruction and raised intracranial pressure in children with craniosynostosis. Eur Respir J 1997;10(2):367-375

3 Taylor WJ, Hayward RD, Lasjaunias P, et al. Enigma of raised intracranial pressure in patients with complex craniosynostosis: the role of abnormal intracranial venous drainage. J Neurosurg 2001;94(3):377-385 10.3171/jns.2001.94.3.0377
4 Marucci DD, Dunaway DJ, Jones BM, Hayward RD. Raised intracranial pressure in Apert syndrome. Plast Reconstr Surg 2008;122(4): 1162-1168, discussion 1169-1170 10.1097/PRS.0b013e31818458fo

5 Tuite GF, Chong WK, Evanson J, et al. The effectiveness of papilledema as an indicator of raised intracranial pressure in children with craniosynostosis. Neurosurgery 1996;38(2):272-278

6 Tuite GF, Evanson J, Chong WK, et al. The beaten copper cranium: a correlation between intracranial pressure, cranial radiographs, and computed tomographic scans in children with craniosynostosis. Neurosurgery 1996;39(4):691-699

7 Wiegand C, Richards P. Measurement of intracranial pressure in children: a critical review of current methods. Dev Med Child Neurol 2007;49(12):935-941

8 Newman WD, Hollman AS, Dutton GN, Carachi R. Measurement of optic nerve sheath diameter by ultrasound: a means of detecting acute raised intracranial pressure in hydrocephalus. $\mathrm{Br} \mathrm{J}$ Ophthalmol 2002;86(10):1109-1113

9 Driessen C, Bannink N, Lequin M, et al. Are ultrasonography measurements of optic nerve sheath diameter an alternative to funduscopy in children with syndromic craniosynostosis? J Neurosurg Pediatr 2011;8(3):329-334

10 Thompson DA, Liasis A, Hardy S, et al. Prevalence of abnormal pattern reversal visual evoked potentials in craniosynostosis. Plast Reconstr Surg 2006;118(1):184-192 10.1097/01.prs.0000220873.72953.3e

11 Fearon JA, Rhodes J. Pfeiffer syndrome: a treatment evaluation. Plast Reconstr Surg 2009;123(5):1560-1569

12 Cinalli G, Spennato P, Sainte-Rose C, et al. Chiari malformation in craniosynostosis. Childs Nerv Syst 2005;21(10):889-901

13 Cinalli G, Renier D, Sebag G, Sainte-Rose C, Arnaud E, Pierre-Kahn A. Chronic tonsillar herniation in Crouzon's and Apert's syndromes: the role of premature synostosis of the lambdoid suture. J Neurosurg 1995;83(4):575-582

14 Cinalli G, Chumas P, Arnaud E, Sainte-Rose C, Renier D. Occipital remodeling and suboccipital decompression in severe craniosynostosis associated with tonsillar herniation. Neurosurgery 1998;42(1):66-71, discussion 71-73

15 Gault DT, Renier D, Marchac D, Jones BM. Intracranial pressure and intracranial volume in children with craniosynostosis. Plast Reconstr Surg 1992;90(3):377-381

16 Renier D, Sainte-Rose C, Marchac D, Hirsch JF. Intracranial pressure in craniostenosis. J Neurosurg 1982;57(3):370-377 10.3171/ jns.1982.57.3.0370

17 Thompson DN, Harkness W, Jones B, Gonsalez S, Andar U, Hayward R. Subdural intracranial pressure monitoring in craniosynostosis: its role in surgical management. Childs Nerv Syst 1995;11(5): 269-275

18 Sgouros S, Goldin JH, Hockley AD, Wake MJ. Posterior skull surgery in craniosynostosis. Childs Nerv Syst 1996;12(11):727-733

19 Wall SA, Goldin JH, Hockley AD, Wake MJ, Poole MD, Briggs M. Fronto-orbital re-operation in craniosynostosis. Br J Plast Surg 1994;47(3):180-184

20 Cornejo-Roldan LR, Roessler E, Muenke M. Analysis of the mutational spectrum of the FGFR2 gene in Pfeiffer syndrome. Hum Genet 1999;104(5):425-431

21 Muenke M, Schell U, Hehr A, et al. A common mutation in the fibroblast growth factor receptor 1 gene in Pfeiffer syndrome. Nat Genet 1994;8(3):269-274

22 Cohen MM Jr. Pfeiffer syndrome update, clinical subtypes, and guidelines for differential diagnosis. Am J Med Genet 1993; 45(3):300-307

23 Howard TD, Paznekas WA, Green ED, et al. Mutations in TWIST, a basic helix-loop-helix transcription factor, in Saethre-Chotzen syndrome. Nat Genet 1997;15(1):36-41

24 el Ghouzzi V, Le Merrer M, Perrin-Schmitt F, et al. Mutations of the TWIST gene in the Saethre-Chotzen syndrome. Nat Genet 1997; 15(1):42-46

25 Foo R, Guo Y, McDonald-McGinn DM, Zackai EH, Whitaker LA, Bartlett SP. The natural history of patients treated for 
TWIST1-confirmed Saethre-Chotzen syndrome. Plast Reconstr Surg 2009;124(6):2085-2095

26 Woods RH, Ul-Haq E, Wilkie AO, et al. Reoperation for intracranial hypertension in TWIST1-confirmed Saethre-Chotzen syndrome: a 15-year review. Plast Reconstr Surg 2009;123(6):1801-1810

27 Paznekas WA, Cunningham ML, Howard TD, et al. Genetic heterogeneity of Saethre-Chotzen syndrome, due to TWIST and FGFR mutations. Am J Hum Genet 1998;62(6):1370-1380

28 Johnson D, Horsley SW, Moloney DM, et al. A comprehensive screen for TWIST mutations in patients with craniosynostosis identifies a new microdeletion syndrome of chromosome band 7p21.1. Am J Hum Genet 1998;63(5):1282-1293

29 Wong GB, Kakulis EG, Mulliken JB. Analysis of fronto-orbital advancement for Apert, Crouzon, Pfeiffer, and Saethre-Chotzen syndromes. Plast Reconstr Surg 2000;105(7):2314-2323

30 Bartlett SP, Foo R. Discussion. Reoperation for intracranial hypertension in TWIST1-confirmed Saethre-Chotzen syndrome: a 15-year review. Plast Reconstr Surg 2009;123(6):1811-1812

31 Muenke M, Gripp KW, McDonald-McGinn DM, et al. A unique point mutation in the fibroblast growth factor receptor 3 gene (FGFR3) defines a new craniosynostosis syndrome. Am J Hum Genet 1997;60(3):555-564

32 Honnebier MB, Cabiling DS, Hetlinger M, McDonald-McGinn DM, Zackai EH, Bartlett SP. The natural history of patients treated for FGFR3-associated (Muenke-type) craniosynostosis. Plast Reconstr Surg 2008;121(3):919-931

33 Cassileth LB, Bartlett SP, Glat PM, et al. Clinical characteristics of patients with unicoronal synostosis and mutations of fibroblast growth factor receptor 3: a preliminary report. Plast Reconstr Surg 2001;108(7):1849-1854

34 Sabatino G, Di Rocco F, Zampino G, Tamburrini G, Caldarelli M, Di Rocco C. Muenke syndrome. Childs Nerv Syst 2004;20(5):297-301

35 Moloney DM, Wall SA, Ashworth GJ, et al. Prevalence of Pro250Arg mutation of fibroblast growth factor receptor 3 in coronal craniosynostosis. Lancet 1997;349(9058):1059-1062

36 Doherty ES, Lacbawan F, Hadley DW, et al. Muenke syndrome (FGFR3-related craniosynostosis): expansion of the phenotype and review of the literature. Am J Med Genet A 2007;143A (24):3204-3215

37 Thomas GP, Wilkie AO, Richards PG, Wall SA. FGFR3 P250R mutation increases the risk of reoperation in apparent 'nonsyndromic' coronal craniosynostosis. J Craniofac Surg 2005;16 (3):347-352, discussion 353-354

38 Arnaud E, Meneses P, Lajeunie E, Thorne JA, Marchac D, Renier D. Postoperative mental and morphological outcome for nonsyndromic brachycephaly. Plast Reconstr Surg 2002;110(1):6-12, discussion 13

39 Czerwinski M, Kolar JC, Fearon JA. Complex craniosynostosis. Plast Reconstr Surg 2011;128(4):955-961
40 Paige KT, Vega SJ, Kelly CP, et al. Age-dependent closure of bony defects after frontal orbital advancement. Plast Reconstr Surg 2006;118(4):977-984

41 Steinbacher DM, Skirpan J, Puchała J, Bartlett SP. Expansion of the posterior cranial vault using distraction osteogenesis. Plast Reconstr Surg 2011;127(2):792-801

42 White N, Evans M, Dover MS, Noons P, Solanki G, Nishikawa H. Posterior calvarial vault expansion using distraction osteogenesis. Childs Nerv Syst: ChNS: official journal of the International Society for Pediatric Neurosurgery 2009;25(2):231-236

43 Fearon JA. Midterm follow-up of midface distraction. Plast Reconstr Surg 2008;122(2):674-675

44 Bradley JP, Gabbay JS, Taub PJ, et al. Monobloc advancement by distraction osteogenesis decreases morbidity and relapse. Plast Reconstr Surg 2006;118(7):1585-1597

45 McCarthy JG, Schreiber J, Karp N, Thorne CH, Grayson BH. Lengthening the human mandible by gradual distraction. Plast Reconstr Surg 1992;89(1):1-8, discussion 9-10

46 Shetye PR, Boutros S, Grayson BH, McCarthy JG. Midterm followup of midface distraction for syndromic craniosynostosis: a clinical and cephalometric study. Plast Reconstr Surg 2007;120(6): $1621-1632$

47 Kobayashi S, Honda T, Saitoh A, Kashiwa K. Unilateral coronal synostosis treated by internal forehead distraction. J Craniofac Surg 1999;10(6):467-471, discussion 472

48 Davis C, MacFarlane MR, Wickremesekera A. Occipital expansion without osteotomies in Apert syndrome. Childs Nerv Syst: ChNS: official journal of the International Society for Pediatric Neurosurgery 2010;26(11):1543-1548

49 Lauritzen CG, Davis C, Ivarsson A, Sanger C, Hewitt TD. The evolving role of springs in craniofacial surgery: the first 100 clinical cases. Plast Reconstr Surg 2008;121(2):545-554

50 Taylor JA, Maugans TA. Comparison of spring-mediated cranioplasty to minimally invasive strip craniectomy and barrel staving for early treatment of sagittal craniosynostosis. J Craniofac Surg 2011;22(4):1225-1229

51 Greene AK, Mulliken JB, Proctor MR, Rogers GF. Pediatric cranioplasty using particulate calvarial bone graft. Plast Reconstr Surg 2008;122(2):563-571

52 Gilardino MS, Jandali S, Whitaker LA, Bartlett SP. Does the incidence of traumatic brain injury in children increase after craniofrontal surgery? J Craniofac Surg 2011;22(4):1284-1286

53 Arnaud E, Marchac D, Renier D. Reduction of morbidity of the frontofacial monobloc advancement in children by the use of internal distraction. Plast Reconstr Surg 2007;120(4):1009-1026

54 Ponniah AJ, Witherow H, Richards R, Evans R, Hayward R, Dunaway D. Three-dimensional image analysis of facial skeletal changes after monobloc and bipartition distraction. Plast Reconstr Surg 2008;122(1):225-231 